\title{
Universal Convergence of Semimeasures on Individual Random SEQUEnCES*
}

\author{
Marcus Hutter \\ IDSIA, Galleria 2, CH-6928 Manno-Lugano, Switzerland \\ marcus@idsia.ch http://www.idsia.ch/ marcus
}

\begin{abstract}
Andrej Muchnik
Institute of New Technologies, 10 Nizhnyaya Radischewskaya

Moscow 109004, Russia_ muchnik@lpcs.math.msu.ru
\end{abstract}

February 1, 2008

\begin{abstract}
Solomonoff's central result on induction is that the posterior of a universal semimeasure $M$ converges rapidly and with probability 1 to the true sequence generating posterior $\mu$, if the latter is computable. Hence, $M$ is eligible as a universal sequence predictor in case of unknown $\mu$. Despite some nearby results and proofs in the literature, the stronger result of convergence for all (Martin-Löf) random sequences remained open. Such a convergence result would be particularly interesting and natural, since randomness can be defined in terms of $M$ itself. We show that there are universal semimeasures $M$ which do not converge for all random sequences, i.e. we give a partial negative answer to the open problem. We also provide a positive answer for some non-universal semimeasures. We define the incomputable measure $D$ as a mixture over all computable measures and the enumerable semimeasure $W$ as a mixture over all enumerable nearly-measures. We show that $W$ converges to $D$ and $D$ to $\mu$ on all random sequences. The Hellinger distance measuring closeness of two distributions plays a central role.
\end{abstract}

\section{Keywords}

Sequence prediction; Algorithmic Information Theory; universal enumerable semimeasure; mixture distributions; posterior convergence; Martin-Löf randomness; quasimeasures.

${ }^{*}$ This work was partially supported by the Swiss National Science Foundation (SNF grant 2100-67712.02) and the Russian Foundation for Basic Research (RFBR grants N04-01-00427 and N02-01-22001). 
Marcus Hutter \& Andrej Muchnik, IDSIA-14-04

\section{Introduction}

A sequence prediction task is defined as to predict the next symbol $x_{n}$ from an observed sequence $x=x_{1} \ldots x_{n-1}$. The key concept to attack general prediction problems is Occam's razor, and to a less extent Epicurus' principle of multiple explanations. The former/latter may be interpreted as to keep the simplest/all theories consistent with the observations $x_{1} \ldots x_{n-1}$ and to use these theories to predict $x_{n}$. Solomonoff Sol64, Sol78 formalized and combined both principles in his universal prior $M$ which assigns high/low probability to simple/complex environments $x$, hence implementing Occam and Epicurus. Formally it is a mixture of all enumerable semimeasures. An abstract characterization of $M$ by Levin [ZL70] is that $M$ is a universal enumerable semimeasure in the sense that it multiplicatively dominates all enumerable semimeasures.

Solomonoff's Sol78] central result is that if the probability $\mu\left(x_{n} \mid x_{1} \ldots x_{n-1}\right)$ of observing $x_{n}$ at time $n$, given past observations $x_{1} \ldots x_{n-1}$ is a computable function, then the universal posterior $M_{n}:=M\left(x_{n} \mid x_{1} \ldots x_{n-1}\right)$ converges (rapidly!) with $\mu$ probability 1 (w.p.1) for $n \rightarrow \infty$ to the true posterior $\mu_{n}:=\mu\left(x_{n} \mid x_{1} \ldots x_{n-1}\right)$, hence $M$ represents a universal predictor in case of unknown "true" distribution $\mu$. Convergence of $M_{n}$ to $\mu_{n}$ w.p.1 tells us that $M_{n}$ is close to $\mu_{n}$ for sufficiently large $n$ for almost all sequences $x_{1} x_{2} \ldots$. It says nothing about whether convergence is true for any particular sequence (of measure 0 ).

Martin-Löf (M.L.) randomness is the standard notion for randomness of individual sequences [ML66, LV97]. A M.L.-random sequence passes all thinkable effective randomness tests, e.g. the law of large numbers, the law of the iterated logarithm, etc. In particular, the set of all $\mu$-random sequences has $\mu$-measure 1 . It is natural to ask whether $M_{n}$ converges to $\mu_{n}$ (in difference or ratio) individually for all M.L.-random sequences. Clearly, Solomonoff's result shows that convergence may at most fail for a set of sequences with $\mu$-measure zero. A convergence result for M.L.-random sequences would be particularly interesting and natural in this context, since M.L.-randomness can be defined in terms of $M$ itself [Lev73]. Despite several attempts to solve this problem [Vov87, VL00, Hut03b, it remained open Hut03c.

In this paper we construct an M.L.-random sequence and show the existence of a universal semimeasure which does not converge on this sequence, hence answering the open question negatively for some $M$. It remains open whether there exist (other) universal semimeasures, probably with particularly interesting additional structure and properties, for which M.L.-convergence holds. The main positive contribution of this work is the construction of a non-universal enumerable semimeasure $W$ which M.L.-converges to $\mu$ as desired. As an intermediate step we consider the incomputable measure $\hat{D}$, defined as a mixture over all computable measures. We show posterior M.L.-convergence of $W$ to $\hat{D}$ and of $\hat{D}$ to $\mu$. The Hellinger distance measuring closeness of two posterior distributions plays a central role in this work.

The paper is organized as follows: In Section 2] we give basic notation and 
results (for strings, numbers, sets, functions, asymptotics, computability concepts, prefix Kolmogorov complexity), and define and discuss the concepts of (universal) (enumerable) (semi)measures. Section 3 summarizes Solomonoff's and Gács' results on posterior convergence of $M$ to $\mu$ with probability 1 . Both results can be derived from a bound on the expected Hellinger sum. We present an improved bound on the expected exponentiated Hellinger sum, which implies very strong assertions on the convergence rate. In Section 4 we investigate whether convergence for all Martin-Löf random sequences hold. We construct a universal semimeasure $M$ and an $\mu$-M.L.random sequence on which $M$ does not converge to $\mu$ for some computable $\mu$. In Section 5 we present our main positive result. We derive a finite bound on the Hellinger sum between $\mu$ and $\hat{D}$, which is exponential in the randomness deficiency of the sequence and double exponential in the complexity of $\mu$. This implies that the posterior of $\hat{D}$ M.L.-converges to $\mu$. Finally, in Section 6 we show that $W$ is nonuniversal and asymptotically M.L.-converges to $\hat{D}$. Section [7] contains discussion and outlook.

\section{Notation \& Universal Semimeasures $M$}

Strings. Let $i, k, n, t \in \mathbb{N}=\{1,2,3, \ldots\}$ be natural numbers, $x, y, z \in \mathcal{X}^{*}=\bigcup_{n=0}^{\infty} \mathcal{X}^{n}$ be finite strings of symbols over finite alphabet $\mathcal{X} \ni a, b$. We denote strings $x$ of length $\ell(x)=n$ by $x=x_{1} x_{2} \ldots x_{n} \in \mathcal{X}^{n}$ with $x_{t} \in \mathcal{X}$ and further abbreviate $x_{k: n}:=$ $x_{k} x_{k+1} \ldots x_{n-1} x_{n}$ for $k \leq n$, and $x_{<n}:=x_{1} \ldots x_{n-1}$, and $\epsilon=x_{<1}=x_{n+1: n} \in \mathcal{X}^{0}=\{\epsilon\}$ for the empty string. Let $\omega=x_{1: \infty} \in \mathcal{X}^{\infty}$ be a generic and $\alpha \in \mathcal{X}^{\infty}$ a specific infinite sequence. For a given sequence $x_{1: \infty}$ we say that $x_{t}$ is on-sequence and $\bar{x}_{t} \neq x_{t}$ is off-sequence. $x_{t}^{\prime}$ may be on- or off-sequence. We identify strings with natural numbers (including zero, $\mathcal{X}^{*} \cong \mathbb{I} \cup\{0\}$ ).

Sets and functions. $\mathbb{Q}, \mathbb{R}, \mathbb{R}_{+}:=[0, \infty)$ are the sets of fractional, real, and nonnegative real numbers, respectively. $\# \mathcal{S}$ denotes the number of elements in set $\mathcal{S}$, $\ln ()$ the natural and $\log ()$ the binary logarithm.

Asymptotics. We abbreviate $\lim _{n \rightarrow \infty}[f(n)-g(n)]=0$ by $f(n) \stackrel{n \rightarrow \infty}{\longrightarrow} g(n)$ and say $f$ converges to $g$, without implying that $\lim _{n \rightarrow \infty} g(n)$ itself exists. We write $f(x) \stackrel{\times}{\leq} g(x)$ for $f(x)=O(g(x))$ and $f(x) \stackrel{ \pm}{\leq} g(x)$ for $f(x) \leq g(x)+O(1)$.

Computability. A function $f: \mathcal{S} \rightarrow \mathbb{R} \cup\{\infty\}$ is said to be enumerable (or lower semicomputable) if the set $\{(x, y): y<f(x), x \in \mathcal{S}, y \in \mathbb{Q}\}$ is recursively enumerable. $f$ is co-enumerable (or upper semi-computable) if $[-f]$ is enumerable. $f$ is computable (or estimable or recursive) if $f$ and $[-f]$ are enumerable. $f$ is approximable (or limitcomputable) if there is a computable function $g: \mathcal{S} \times \mathbb{N} \rightarrow \mathbb{R}$ with $\lim _{n \rightarrow \infty} g(x, n)=$ $f(x)$. The set of enumerable functions is recursively enumerable.

Complexity. The conditional prefix (Kolmogorov) complexity $K(x \mid y):=\min \{\ell(p)$ : $U(y, p)=x$ halts $\}$ is the length of the shortest binary program $p \in\{0,1\}^{*}$ on a universal prefix Turing machine $U$ with output $x \in \mathcal{X}^{*}$ and input $y \in \mathcal{X}^{*}$ [LV97]. $K(x):=K(x \mid \epsilon)$. For non-string objects $o$ we define $K(o):=K(\langle o\rangle)$, where $\langle o\rangle \in \mathcal{X}^{*}$ is some standard 
code for $o$. In particular, if $\left(f_{i}\right)_{i=1}^{n}$ is an enumeration of all enumerable functions, we define $K\left(f_{i}\right)=K(i)$. We only need the following elementary properties: The co-enumerability of $K$, the upper bounds $K(x \mid \ell(x)) \stackrel{ \pm}{\leq}(x) \log |\mathcal{X}|$ and $K(n) \stackrel{ \pm}{\leq} \log n$, and $K(x \mid y) \stackrel{ \pm}{\leq} K(x)$, subadditivity $K(x) \stackrel{ \pm}{\leq} K(x, y) \stackrel{ \pm}{\leq} K(y)+K(x \mid y)$, and information non-increase $K(f(x)) \stackrel{ \pm}{\leq}(x)+K(f)$ for recursive $f: \mathcal{X}^{*} \rightarrow \mathcal{X}^{*}$.

We need the concepts of (universal) (semi)measures for strings [ZL70].

Definition 1 ((Semi)measures) We call $\nu: \mathcal{X}^{*} \rightarrow[0,1]$ a semimeasure if $\nu(x) \geq$ $\sum_{a \in \mathcal{X}} \nu(x a) \forall x \in \mathcal{X}^{*}$, and a (probability) measure if equality holds and $\nu(\epsilon)=1 . \nu(x)$ denotes the $\nu$-probability that a sequence starts with string $x$. Further, $\nu(a \mid x):=\frac{\nu(x a)}{\nu(x)}$ is the posterior $\nu$-probability that the next symbol is $a \in \mathcal{X}$, given sequence $x \in \mathcal{X}^{*}$.

Definition 2 (Universal semimeasures $M$ ) A semimeasure $M$ is called a universal element of a class of semimeasures $\mathcal{M}$, if

$$
M \in \mathcal{M} \text { and } \forall \nu \in \mathcal{M} \exists w_{\nu}>0: M(x) \geq w_{\nu} \cdot \nu(x) \forall x \in \mathcal{X}^{*} .
$$

From now on we consider the (in a sense) largest class $\mathcal{M}$ which is relevant from a constructive point of view (but see Sch02, Hut03b] for even larger constructive classes), namely the class of all semimeasures, which can be enumerated (=effectively be approximated) from below:

$$
\mathcal{M}:=\text { class of all enumerable semimeasures. }
$$

Solomonoff [Sol64, Eq.(7)] defined the universal posterior $M(x \mid y)=M(x y) / M(y)$ with $M(x)$ defined as the probability that the output of a universal monotone Turing machine starts with $x$ when provided with fair coin flips on the input tape. Levin [ZL70] has shown that this $M$ is a universal enumerable semimeasure. Another possible definition of $M$ is as a (Bayes) mixture [Sol64, ZL70, Sol78, LV97, Hut03b]: $\tilde{M}(x)=\sum_{\nu \in \mathcal{M}} 2^{-K(\nu)} \nu(x)$, where $K(\nu)$ is the length of the shortest program computing function $\nu$. Levin [ZL70] has shown that the class of all enumerable semimeasures is enumerable (with repetitions), hence $\tilde{M}$ is enumerable, since $K$ is co-enumerable. Hence $\tilde{M} \in \mathcal{M}$, which implies

$$
M(x) \geq w_{\tilde{M}} \tilde{M}(x) \geq w_{\tilde{M}} 2^{-K(\nu)} \nu(x)=w_{\nu}^{\prime} \nu(x), \quad \text { where } \quad w_{\nu}^{\prime} \stackrel{x}{=} 2^{-K(\nu)} .
$$

Up to a multiplicative constant, $M$ assigns higher probability to all $x$ than any other enumerable semimeasure. All $M$ have the same very slowly decreasing (in $\nu$ ) domination constants $w_{\nu}^{\prime}$, essentially because $M \in \mathcal{M}$. We drop the prime from $w_{\nu}^{\prime}$ in the following. The mixture definition $\tilde{M}$ immediately generalizes to arbitrary weighted sums of (semi)measures over other countable classes than $\mathcal{M}$, but the class may not contain the mixture, and the domination constants may be rapidly decreasing. We will exploit this for the construction of the non-universal semimeasure $W$ in Sections 5 and 6 . 


\section{Posterior Convergence with Probability 1}

The following convergence results for $M$ are well-known [Sol78, LV97, Hut03a].

Theorem 3 (Convergence of $M$ to $\mu$ w.p.1) For any universal semimeasure $M$ and any computable measure $\mu$ it holds:

$$
M\left(x_{n}^{\prime} \mid x_{<n}\right) \rightarrow \mu\left(x_{n}^{\prime} \mid x_{<n}\right) \text { for any } x_{n}^{\prime} \text { and } \frac{M\left(x_{n} \mid x_{<n}\right)}{\mu\left(x_{n} \mid x_{<n}\right)} \rightarrow 1 \text {, both w.p.1 for } n \rightarrow \infty \text {. }
$$

The first convergence in difference is Solomonoff's Sol78] celebrated convergence result. The second convergence in ratio has first been derived by Gács [LV97]. Note the subtle difference between the two convergence results. For any sequence $x_{1: \infty}^{\prime}$ (possibly constant and not necessarily random), $M\left(x_{n}^{\prime} \mid x_{<n}\right)-\mu\left(x_{n}^{\prime} \mid x_{<n}\right)$ converges to zero w.p.1 (referring to $x_{1: \infty}$ ), but no statement is possible for $M\left(x_{n}^{\prime} \mid x_{<n}\right) / \mu\left(x_{n}^{\prime} \mid x_{<n}\right)$, since $\liminf \mu\left(x_{n}^{\prime} \mid x_{<n}\right)$ could be zero. On the other hand, if we stay on-sequence $\left(x_{1: \infty}^{\prime}=x_{1: \infty}\right)$, we have $M\left(x_{n} \mid x_{<n}\right) / \mu\left(x_{n} \mid x_{<n}\right) \rightarrow 1$ (whether $\inf \mu\left(x_{n} \mid x_{<n}\right)$ tends to zero or not does not matter). Indeed, it is easy to give an example where $M\left(x_{n}^{\prime} \mid x_{<n}\right) / \mu\left(x_{n}^{\prime} \mid x_{<n}\right)$ diverges. For $\mu\left(1 \mid x_{<n}\right)=1-\mu\left(0 \mid x_{<n}\right)=\frac{1}{2} n^{-3}$ we get $\mu\left(0_{1: n}\right)=\prod_{t=1}^{n}\left(1-\frac{1}{2} t^{-3}\right) \stackrel{n \rightarrow \infty}{\longrightarrow} c=0.450 \ldots>0$, i.e. $0_{1: \infty}$ is $\mu$-random. On the other hand, one can show that $M\left(0_{<n}\right)=O(1)$ and $M\left(0_{<n} 1\right) \stackrel{\times}{=} 2^{-K(n)}$, which implies $\frac{M\left(1 \mid 0_{<n}\right)}{\mu\left(1 \mid 0_{<n}\right)} \stackrel{\times}{=} n^{3} \cdot 2^{-K(n)} \stackrel{\times}{\geq} n \rightarrow \infty$ for $n \rightarrow \infty(K(n) \stackrel{ \pm}{\leq} 2 \log n)$.

Theorem 3 follows from (the discussion after) Lemma 4 due to $M(x) \geq w_{\mu} \mu(x)$. Actually the Lemma strengthens and generalizes Theorem 3. In the following we denote expectations w.r.t. measure $\rho$ by $\mathbf{E}_{\rho}$, i.e. for a function $f: \mathcal{X}^{n} \rightarrow \mathbb{R}, \mathbf{E}_{\rho}[f]=$ $\sum_{x_{1: n}}^{\prime} \rho\left(x_{1: n}\right) f\left(x_{1: n}\right)$, where $\sum^{\prime}$ sums over all $x_{1: n}$ for which $\rho\left(x_{1: n}\right) \neq 0$. Using $\Sigma^{\prime}$ instead $\sum$ is important for partial functions $f$ undefined on a set of $\rho$-measure zero. Similarly $\mathbf{P}_{\rho}$ denotes the $\rho$-probability.

Lemma 4 (Expected Bounds on Hellinger Sum) Let $\mu$ be a measure and $\nu$ be a semimeasure with $\nu(x) \geq w \cdot \mu(x) \forall x$. Then the following bounds on the Hellinger distance $h_{t}\left(\nu, \mu \mid \omega_{<t}\right):=\sum_{a \in \mathcal{X}}\left(\sqrt{\nu\left(a \mid \omega_{<t}\right)}-\sqrt{\mu\left(a \mid \omega_{<t}\right)}\right)^{2}$ hold:

$$
\sum_{t=1}^{\infty} \mathbf{E}\left[\left(\sqrt{\frac{\nu\left(\omega_{t} \mid \omega_{<t}\right)}{\mu\left(\omega_{t} \mid \omega_{<t}\right)}}-1\right)^{2}\right] \stackrel{(i)}{\leq} \sum_{t=1}^{\infty} \mathbf{E}\left[h_{t}\right] \stackrel{(i i)}{\leq} 2 \ln \left\{\mathbf{E}\left[\exp \left(\frac{1}{2} \sum_{t=1}^{\infty} h_{t}\right)\right]\right\} \stackrel{(i i i)}{\leq} \ln w^{-1}
$$

where $\mathbf{E}$ means expectation w.r.t. $\mu$.

The $\ln w^{-1}$-bounds on the first and second expression have first been derived in Hut03a, the second being a variation of Solomonoff's bound $\sum_{n} \mathbf{E}\left[\left(\nu\left(0 \mid x_{<n}\right)-\right.\right.$ $\left.\left.\mu\left(0 \mid x_{<n}\right)\right)^{2}\right] \leq \frac{1}{2} \ln w^{-1}$. If sequence $x_{1} x_{2} \ldots$ is sampled from the probability measure $\mu$, these bounds imply

$$
\nu\left(x_{n}^{\prime} \mid x_{<n}\right) \rightarrow \mu\left(x_{n}^{\prime} \mid x_{<n}\right) \text { for any } x_{n}^{\prime} \text { and } \frac{\nu\left(x_{n} \mid x_{<n}\right)}{\mu\left(x_{n} \mid x_{<n}\right)} \rightarrow 1 \text {, both w.p.1 for } n \rightarrow \infty
$$


where w.p.1 stands here and in the following for 'with $\mu$-probability 1 '.

Convergence is "fast" in the following sense: The second bound $\left(\sum_{t} \mathbf{E}\left[h_{t}\right] \leq \ln w^{-1}\right)$ implies that the expected number of times $t$ in which $h_{t} \geq \varepsilon$ is finite and bounded by $\frac{1}{\varepsilon} \ln w^{-1}$. The new third bound represents a significant improvement. It implies by means of a Markov inequality that the probability of even only marginally exceeding this number is extremely small, and that $\sum_{t} h_{t}$ is very unlikely to exceed $\ln w^{-1}$ by much. More precisely:

$$
\begin{gathered}
\mathbf{P}\left[\#\left\{t: h_{t} \geq \varepsilon\right\} \geq \frac{1}{\varepsilon}\left(\ln w^{-1}+c\right)\right] \leq \mathbf{P}\left[\sum_{t} h_{t} \geq \ln w^{-1}+c\right] \\
=\mathbf{P}\left[\exp \left(\frac{1}{2} \sum_{t} h_{t}\right) \geq \mathrm{e}^{c / 2} w^{-1 / 2}\right] \leq \sqrt{w} \mathbf{E}\left[\exp \left(\frac{1}{2} \sum_{t} h_{t}\right)\right] \mathrm{e}^{-c / 2} \leq \mathrm{e}^{-c / 2}
\end{gathered}
$$

Proof. We use the abbreviations $\rho_{t}=\rho\left(x_{t} \mid x_{<t}\right)$ and $\rho_{1: n}=\rho_{1} \cdot \ldots \cdot \rho_{n}=\rho\left(x_{1: n}\right)$ for $\rho \in\{\mu, \nu, R, N, \ldots\}$ and $h_{t}=\sum_{x_{t}}\left(\sqrt{\nu_{t}}-\sqrt{\mu_{t}}\right)^{2}$.

(i) follows from

$$
\mathbf{E}\left[\left(\sqrt{\frac{\nu_{t}}{\mu_{t}}}-1\right)^{2} \mid x_{<t}\right] \equiv \sum_{x_{t}: \mu_{t} \neq 0} \mu_{t}\left(\sqrt{\frac{\nu_{t}}{\mu_{t}}}-1\right)^{2}=\sum_{x_{t}: \mu_{t} \neq 0}\left(\sqrt{\nu_{t}}-\sqrt{\mu_{t}}\right)^{2} \leq h_{t}
$$

by taking the expectation $\mathbf{E}[]$ and sum $\sum_{t=1}^{\infty}$.

(ii) follows from Jensen's inequality $\exp (\mathbf{E}[f]) \leq \mathbf{E}[\exp (f)]$ for $f=\frac{1}{2} \sum_{t} h_{t}$.

(iii) We exploit a construction used in [Vov87, Thm.1]. For discrete (semi)measures $p$ and $q$ with $\sum_{i} p_{i}=1$ and $\sum_{i} q_{i} \leq 1$ it holds:

$$
\sum_{i} \sqrt{p_{i} q_{i}} \leq 1-\frac{1}{2} \sum_{i}\left(\sqrt{p_{i}}-\sqrt{q_{i}}\right)^{2} \leq \exp \left[-\frac{1}{2} \sum_{i}\left(\sqrt{p_{i}}-\sqrt{q_{i}}\right)^{2}\right]
$$

The first inequality is obvious after multiplying out the second expression. The second inequality follows from $1-x \leq \mathrm{e}^{-x}$. Vovk [Vov87] defined a measure $R_{t}:=$ $\sqrt{\mu_{t} \nu_{t}} / N_{t}$ with normalization $N_{t}:=\sum_{x_{t}} \sqrt{\mu_{t} \nu_{t}}$. Applying (3) for measure $\mu$ and semimeasure $\nu$ we get $N_{t} \leq \exp \left(-\frac{1}{2} h_{t}\right)$. Together with $\nu(x) \geq w \cdot \mu(x) \forall x$ this implies

$$
\prod_{t=1}^{n} R_{t}=\prod_{t=1}^{n} \frac{\sqrt{\mu_{t} \nu_{t}}}{N_{t}}=\frac{\sqrt{\mu_{1: n} \nu_{1: n}}}{N_{1: n}}=\mu_{1: n} \sqrt{\frac{\nu_{1: n}}{\mu_{1: n}}} N_{1: n}^{-1} \geq \mu_{1: n} \sqrt{w} \exp \left(\frac{1}{2} \sum_{t=1}^{n} h_{t}\right) .
$$

Summing over $x_{1: n}$ and exploiting $\sum_{x_{t}} R_{t}=1$ we get $1 \geq \sqrt{w} \mathbf{E}\left[\exp \left(\frac{1}{2} \sum_{t} h_{t}\right)\right]$, which proves $($ iii).

The bound and proof may be generalized to $1 \geq w^{\kappa} \mathbf{E}\left[\exp \left(\frac{1}{2} \sum_{t} \sum_{x_{t}}\left(\nu_{t}^{\kappa}-\mu_{t}^{\kappa}\right)^{1 / \kappa}\right)\right]$ with $0 \leq \kappa \leq \frac{1}{2}$ by defining $R_{t}=\mu_{t}^{1-\kappa} \nu_{t}^{\kappa} / N_{t}$ with $N_{t}=\sum_{x_{t}} \mu_{t}^{1-\kappa} \nu_{t}^{\kappa}$ and exploiting $\sum_{i} p_{i}^{1-\kappa} q_{i}^{\kappa} \leq \exp \left(-\frac{1}{2} \sum_{i}\left(p_{i}^{\kappa}-q_{i}^{\kappa}\right)^{1 / \kappa}\right)$.

One can show that the constant $\frac{1}{2}$ in Lemma 4 can essentially not been improved. Increasing it to a constant $\alpha>1$ makes the expression infinite for some (Bernoulli) distribution $\mu$ (however we choose $\nu$ ). For $\nu=M$ the expression can become already infinite for $\alpha>\frac{1}{2}$ and some computable measure $\mu$. 


\section{Non-Convergence in Martin-Löf Sense}

Convergence of $M\left(x_{n} \mid x_{<n}\right)$ to $\mu\left(x_{n} \mid x_{<n}\right)$ with $\mu$-probability 1 tells us that $M\left(x_{n} \mid x_{<n}\right)$ is close to $\mu\left(x_{n} \mid x_{<n}\right)$ for sufficiently large $n$ on "most" sequences $x_{1: \infty}$. It says nothing whether convergence is true for any particular sequence (of measure 0). Martin-Löf randomness can be used to capture convergence properties for individual sequences. Martin-Löf randomness is a very important concept of randomness of individual sequences, which is closely related to Kolmogorov complexity and Solomonoff's universal semimeasure $M$. Levin gave a characterization equivalent to Martin-Löf's original definition [Lev73]:

Definition 5 (Martin-Löf random sequences) $A$ sequence $\omega=\omega_{1: \infty}$ is $\mu$ Martin-Löf random (M.M.L.) iff there is a constant $c<\infty$ such that $M\left(\omega_{1: n}\right) \leq$ $c \cdot \mu\left(\omega_{1: n}\right)$ for all $n$. Moreover, $d_{\mu}(\omega):=\sup _{n}\left\{\log \frac{M\left(\omega_{1: n}\right)}{\mu\left(\omega_{1: n}\right)}\right\} \leq \log c$ is called the randomness deficiency of $\omega$.

One can show that an M.L.-random sequence $x_{1: \infty}$ passes all thinkable effective randomness tests, e.g. the law of large numbers, the law of the iterated logarithm, etc. In particular, the set of all $\mu$.M.L.-random sequences has $\mu$-measure 1.

The open question we study in this section is whether $M$ converges to $\mu$ (in difference or ratio) individually for all Martin-Löf random sequences. Clearly, Theorem 3 implies that convergence $\mu$.M.L. may at most fail for a set of sequences with $\mu$-measure zero. A convergence M.L. result would be particularly interesting and natural for $M$, since M.L.-randomness can be defined in terms of $M$ itself (Definition 5).

The state of the art regarding this problem may be summarized as follows: Vov87 contains a (non-improvable?) result which is slightly too weak to imply M.L.-convergence, [LV97, Thm.5.2.2] and [VL00, Thm.10] contain an erroneous proof for M.L.-convergence, and Hut03b proves a theorem indicating that the answer may be hard and subtle (see Hut03b for details).

The main contribution of this section is a partial answer to this question. We show that M.L.-convergence fails at least for some universal semimeasures:

Theorem 6 (Universal semimeasure non-convergence) There exists a universal semimeasure $M$ and a computable measure $\mu$ and a $\mu$.M.L.-random sequence $\alpha$, such that $\quad M\left(\alpha_{n} \mid \alpha_{<n}\right) \nrightarrow \mu\left(\alpha_{n} \mid \alpha_{<n}\right) \quad$ for $n \rightarrow \infty$.

This implies that also $M_{n} / \mu_{n}$ does not converge (since $\mu_{n} \leq 1$ is bounded). We do not know whether Theorem [ 6 holds for all universal semimeasures. The proof idea is to construct an enumerable (semi)measure $\nu$ such that $\nu$ dominates $M$ on some $\mu$-random sequence $\alpha$, but $\nu\left(\alpha_{n} \mid \alpha_{<n}\right) \nmid \mu\left(\alpha_{n} \mid \alpha_{<n}\right)$. Then we mix $M$ to $\nu$ to make $\nu$ universal, but with larger contribution from $\nu$, in order to preserve non-convergence. There is also non-constructive proof showing that an arbitrary small contamination with $\nu$ can lead to non-convergence. We only present the constructive proof. 
Proof. We consider binary alphabet $\mathcal{X}=\{0,1\}$ only. Let $\mu(x)=\lambda(x):=2^{-\ell(x)}$ be the uniform measure. We define the sequence $\alpha$ as the (in a sense) lexicographically first (or equivalently left-most in the tree of sequences) $\lambda$. M.L.-random sequence. Formally we define $\alpha$, inductively in $n=1,2,3, \ldots$ by

$$
\alpha_{n}=0 \text { if } M\left(\alpha_{<n} 0\right) \leq 2^{-n} \text {, and } \alpha_{n}=1 \text { else. }
$$

We know that $M(\epsilon) \leq 1$ and $M\left(\alpha_{<n} 0\right) \leq 2^{-n}$ if $\alpha_{n}=0$. Inductively, assuming $M\left(\alpha_{<n}\right) \leq 2^{-n+1}$ for $\alpha_{n}=1$ we have $2^{-n+1} \geq M\left(\alpha_{<n}\right) \geq M\left(\alpha_{<n} 0\right)+M\left(\alpha_{<n} 1\right) \geq$ $2^{-n}+M\left(\alpha_{<n} 1\right)$ since $M$ is a semimeasure, hence $M\left(\alpha_{<n} 1\right) \leq 2^{-n}$. Hence

$$
M\left(\alpha_{1: n}\right) \leq 2^{-n} \equiv \lambda\left(\alpha_{1: n}\right) \forall n \text {, i.e. } \alpha \text { is } \lambda \text {.M.L.-random. }
$$

Let $M^{t}$ with $t=1,2,3, \ldots$ be computable approximations of $M$, which enumerate $M$, i.e. $M^{t}(x) \nearrow M(x)$ for $t \rightarrow \infty$. W define $\alpha^{t}$ like $\alpha$ but with $M$ replaced by $M^{t}$ in the definition. $M^{t} \nearrow M$ implies $\alpha^{t} \nearrow \alpha$ (lexicographically increasing). We define an enumerable semimeasure $\nu$ as follows:

$$
\nu^{t}(x):=\left\{\begin{array}{cll}
2^{-t} & \text { if } \ell(x)=t \text { and } x<\alpha_{1: t}^{t} \\
0 & \text { if } \ell(x)=t \text { and } x \geq \alpha_{1: t}^{t} \\
0 & \text { if } \ell(x)>t \\
\nu^{t}(x 0)+\nu^{t}(x 1) & \text { if } \ell(x)<t
\end{array}\right.
$$

where $<$ is the lexicographical ordering on sequences. $\nu^{t}$ is a semimeasure, and with $\alpha^{t}$ also $\nu^{t}$ is computable and monotone increasing in $t$, hence $\nu:=\lim _{t \rightarrow \infty} \nu^{t}$ is an enumerable semimeasure (indeed, $\frac{\nu(x)}{\nu(\epsilon)}$ is a measure). We could have defined a $\nu_{t n}$ by replacing $\alpha_{1: t}^{t}$ with $\alpha_{1: t}^{n}$ in (6). Since $\nu_{t n}$ is monotone increasing in $t$ and $n$, any order of $t, n \rightarrow \infty$ leads to $\nu$, so we have chosen arbitrarily $t=n$. By induction (starting from $\ell(x)=t)$ it follows that

$$
\nu^{t}(x)=2^{-\ell(x)} \quad \text { if } \quad x<\alpha_{1: \ell(x)}^{t} \quad \text { and } \quad \ell(x) \leq t, \quad \nu^{t}(x)=0 \quad \text { if } \quad x>\alpha_{1: \ell(x)}^{t}
$$

On-sequence, i.e. for $x=\alpha_{1: n}, \nu^{t}$ is somewhere in-between 0 and $2^{-\ell(x)}$. Since sequence $\alpha:=\lim _{t} \alpha^{t}$ is $\lambda$.M.L.-random it contains 01 infinitely often, actually $\alpha_{n} \alpha_{n+1}=01$ for a non-vanishing fraction of $n$. In the following we fix such an $n$. For $t \geq n$ we get

$$
\nu^{t}\left(\alpha_{<n}\right)=\nu^{t}\left(\alpha_{<n} 0\right)+\nu^{t}(\underbrace{\alpha_{<n} 1}_{>\alpha_{1: n} \geq \alpha_{1: n}^{t}, \text { since } \alpha_{n}=0})=\nu^{t}\left(\alpha_{<n} 0\right)=\nu^{t}\left(\alpha_{1: n}\right) \quad \Rightarrow \quad \nu\left(\alpha_{<n}\right)=\nu\left(\alpha_{1: n}\right)
$$

This ensures $\nu\left(\alpha_{n} \mid \alpha_{<n}\right)=1 \neq \frac{1}{2}=\lambda_{n}$. For $t>n$ large enough such that $\alpha_{1: n+1}^{t}=\alpha_{1: n+1}$ we get:

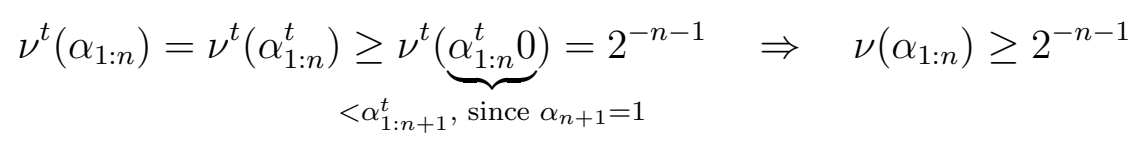


This ensures $\nu\left(\alpha_{1: n}\right) \geq 2^{-n-1} \geq \frac{1}{2} M\left(\alpha_{1: n}\right)$ by (15). Let $M$ be any universal semimeasure and $0<\gamma<\frac{1}{5}$. Then $M^{\prime}(x):=(1-\gamma) \nu(x)+\gamma M(x) \forall x$ is also a universal semimeasure with

$$
\begin{gathered}
M\left(\alpha_{<n}\right) \leq 2^{-n+1} \text { and } M\left(\alpha_{1: n}\right) \geq 0 \\
M^{\prime}\left(\alpha_{n} \mid \alpha_{<n}\right)=\frac{(1-\gamma) \nu\left(\alpha_{1: n}\right)+\gamma M\left(\alpha_{1: n}\right)}{(1-\gamma) \nu\left(\alpha_{<n}\right)+\gamma M\left(\alpha_{<n}\right)} \geq \frac{(1-\gamma) \nu\left(\alpha_{1: n}\right)}{(1-\gamma) \nu\left(\alpha_{<n}\right)+\gamma 2^{-n+1}} \\
=\frac{1-\gamma}{1-\gamma+\gamma 2^{-n+1} / \nu\left(\alpha_{1: n}\right)} \geq \frac{1-\gamma}{1+3 \gamma}>\frac{1}{2} . \\
\quad \begin{array}{c}
\uparrow\left(\alpha_{1: n}\right) \geq 2^{-n-1}
\end{array}
\end{gathered}
$$

For instance for $\gamma=\frac{1}{9}$ we have $M^{\prime}\left(\alpha_{n} \mid \alpha_{<n}\right) \geq \frac{2}{3} \neq \frac{1}{2}=\lambda\left(\alpha_{n} \mid \alpha_{<n}\right)$ for a non-vanishing fraction of $n$ 's.

A converse of Theorem [6] can also be shown:

Theorem 7 (Convergence on non-random sequences) For every universal semimeasure $M$ there exist computable measures $\mu$ and non- $\mu$.M.L.-random sequences $\alpha$ for which $M\left(\alpha_{n} \mid \alpha_{<n}\right) / \mu\left(\alpha_{n} \mid \alpha_{<n}\right) \rightarrow 1$.

\section{Convergence in Martin-Löf Sense}

In this and the next section we give a positive answer to the question of posterior M.L.-convergence to $\mu$. We consider general finite alphabet $\mathcal{X}$.

Theorem 8 (Universal predictor for M.L.-random sequences) There exists an enumerable semimeasure $W$ such that for every computable measure $\mu$ and every M.M.L.-random sequence $\omega$, the posteriors converge to each other:

$$
W\left(a \mid \omega_{<t}\right) \stackrel{t \rightarrow \infty}{\longrightarrow} \mu\left(a \mid \omega_{<t}\right) \quad \text { for all } \quad a \in \mathcal{X} \quad \text { if } \quad d_{\mu}(\omega)<\infty .
$$

The semimeasure $W$ we will construct is not universal in the sense of dominating all enumerable semimeasures, unlike $M$. Normalizing $W$ shows that there is also a measure whose posterior converges to $\mu$, but this measure is not enumerable, only approximable. For proving Theorem 8 we first define an intermediate measure $D$ as a mixture over all computable measures, which is not even approximable. Based on Lemmas 4910. Proposition 11] shows that $D$ M.L.-converges to $\mu$. We then define the concept of quasimeasures and an enumerable semimeasure $W$ as a mixture over all enumerable quasimeasures. Proposition 12 shows that $W$ M.L.-converges to $D$. Theorem 8 immediately follows from Propositions [1] and 12.

Lemma 9 (Hellinger Chain) Let $h(p, q):=\sum_{i=1}^{N}\left(\sqrt{p_{i}}-\sqrt{q_{i}}\right)^{2}$ be the Hellinger distance between $p=\left(p_{i}\right)_{i=1}^{N} \in \mathbb{R}_{+}^{N}$ and $q=\left(q_{i}\right)_{i=1}^{N} \in \mathbb{R}_{+}^{N}$. Then 
i) for $p, q, r \in \mathbb{R}_{+}^{N} \quad h(p, q) \leq(1+\beta) h(p, r)+\left(1+\beta^{-1}\right) h(r, q)$, any $\beta>0$

ii) for $p^{1}, \ldots, p^{m} \in \mathbb{R}_{+}^{N} h\left(p^{1}, p^{m}\right) \leq 3 \sum_{k=2}^{m} k^{2} h\left(p^{k-1}, p^{k}\right)$

Proof. (i) For any $x, y \in \mathbb{R}$ and $\beta>0$ we have $(x+y)^{2} \leq(1+\beta) x^{2}+\left(1+\beta^{-1}\right) y^{2}$. Inserting $x=\sqrt{p_{i}}-\sqrt{r_{i}}$ and $y=\sqrt{r_{i}}-\sqrt{q_{i}}$ and summing over $i$ proves $(i)$.

(ii) Apply $(i)$ for the triples $\left(p^{k}, p^{k+1}, p^{m}\right)$ for and in order of $k=1,2, \ldots, m-2$ with $\beta=\beta_{k}=k(k+1)$ and finally use $\prod_{j=1}^{k-2}\left(1+\beta_{j}^{-1}\right) \leq \mathrm{e} \leq 3$.

We need a way to convert expected bounds to bounds on individual M.L. random sequences, sort of a converse of "M.L. implies w.p.1". Consider for instance the Hellinger sum $H(\omega):=\sum_{t=1}^{\infty} h_{t}(\mu, \rho) / \ln w^{-1}$ between two computable measures $\rho \geq$ $w \cdot \mu$. Then $H$ is an enumerable function and Lemma 4 implies $\mathbf{E}[H] \leq 1$, hence $H$ is an integral $\mu$-test. $H$ can be increased to an enumerable $\mu$-submartingale $\bar{H}$. The universal $\mu$-submartingale $M / \mu$ multiplicatively dominates all enumerable submartingales (and hence $\bar{H}$ ). Since $M / \mu \leq 2^{d_{\mu}(\omega)}$, this implies the desired bound $H(\omega) \stackrel{\times}{\leq} 2^{d_{\mu}(\omega)}$ for individual $\omega$. We give a self-contained direct proof, explicating all important constants.

Lemma 10 (Expected to Individual Bound) Let $F(\omega) \geq 0$ be an enumerable function and $\mu$ be an enumerable measure and $\varepsilon>0$ be co-enumerable. Then:

$$
\text { If } \mathbf{E}_{\mu}[F] \leq \varepsilon \quad \text { then } F(\omega) \stackrel{\times}{\leq} \varepsilon \cdot 2^{K\left(\mu, F,{ }^{1} / \varepsilon\right)+d_{\mu}(\omega)} \quad \forall \omega
$$

where $d_{\mu}(\omega)$ is the $\mu$-randomness deficiency of $\omega$ and $K\left(\mu, F,{ }^{1} / \varepsilon\right)$ is the length of the shortest program for $\mu, F$, and $1 / \varepsilon$.

Lemma 10 roughly says that for $\mu, F$, and $\varepsilon \stackrel{\times}{=} \mathbf{E}_{\mu}[F]$ with short program $(K(\mu, F, 1 / \varepsilon)=O(1))$ and $\mu$-random $\omega\left(d_{\mu}(\omega)=O(1)\right)$ we have $F(\omega) \stackrel{x}{\leq} \mathbf{E}_{\mu}[F]$.

Proof. Let $F(\omega)=\lim _{n \rightarrow \infty} F_{n}(\omega)=\sup _{n} F_{n}(\omega)$ be enumerated by an increasing sequence of computable functions $F_{n}(\omega) . F_{n}(\omega)$ can be chosen to depend on $\omega_{1: n}$ only, i.e. $F_{n}(\omega)=F_{n}\left(\omega_{1: n}\right)$ is independent of $\omega_{n+1: \infty}$. Let $\varepsilon_{n} \searrow \varepsilon$ co-enumerate $\varepsilon$. We define

$\bar{\mu}_{n}\left(\omega_{1: k}\right):=\varepsilon_{n}^{-1} \sum_{\omega_{k+1: n} \in \mathcal{X}^{n-k}} \mu\left(\omega_{1: n}\right) F_{n}\left(\omega_{1: n}\right)$ for $k \leq n, \quad$ and $\quad \bar{\mu}_{n}\left(\omega_{1: k}\right)=0$ for $k>n$.

$\bar{\mu}_{n}$ is a computable semimeasure for each $n$ (due to $\mathbf{E}_{\mu}\left[F_{n}\right] \leq \varepsilon$ ) and increasing in $n$, since

$$
\begin{aligned}
& \bar{\mu}_{n}\left(\omega_{1: k}\right) \geq 0=\bar{\mu}_{n-1}\left(\omega_{1: k}\right) \text { for } k \geq n \text { and } \\
& \bar{\mu}_{n}\left(\omega_{<n}\right) \underset{\uparrow}{\geq} \sum_{\omega_{n} \in \mathcal{X}} \varepsilon_{n}^{-1} \mu\left(\omega_{1: n}\right) F_{n-1}\left(\omega_{<n}\right) \underset{\uparrow}{=} \varepsilon_{n}^{-1} \mu\left(\omega_{<n}\right) F_{n-1}\left(\omega_{<n}\right) \underset{\uparrow}{\geq} \bar{\mu}_{n-1}\left(\omega_{<n}\right) \\
& F_{n} \geq F_{n-1} \quad \mu \text { measure } \quad \varepsilon_{n} \leq \varepsilon_{n-1}
\end{aligned}
$$


and similarly for $k<n-1$. Hence $\bar{\mu}:=\bar{\mu}_{\infty}$ is an enumerable semimeasure (indeed $\bar{\mu}$ is proportional to a measure). From dominance (2) we get

$$
M\left(\omega_{1: n}\right) \stackrel{\times}{\geq} 2^{-K(\bar{\mu})} \bar{\mu}\left(\omega_{1: n}\right) \geq 2^{-K(\bar{\mu})} \bar{\mu}_{n}\left(\omega_{1: n}\right)=2^{-K(\bar{\mu})} \varepsilon_{n}^{-1} \mu\left(\omega_{1: n}\right) F_{n}\left(\omega_{1: n}\right) .
$$

In order to enumerate $\bar{\mu}$, we need to enumerate $\mu, F$, and $\varepsilon^{-1}$, hence $K(\bar{\mu}) \stackrel{ \pm}{\leq}(\mu, F, 1 / \varepsilon)$, so we get

$$
F_{n}(\omega) \equiv F_{n}\left(\omega_{1: n}\right) \stackrel{\times}{\leq} \varepsilon_{n} \cdot 2^{K(\mu, F, 1 / \varepsilon)} \cdot \frac{M\left(\omega_{1: n}\right)}{\mu\left(\omega_{1: n}\right)} \leq \varepsilon_{n} \cdot 2^{K(\mu, F, 1 / \varepsilon)+d_{\mu}(\omega)}
$$

Taking the limit $F_{n} \nearrow F$ and $\varepsilon_{n} \searrow \varepsilon$ completes the proof.

Let $\mathcal{M}=\left\{\nu_{1}, \nu_{2}, \ldots\right\}$ be an enumeration of all enumerable semimeasures, $J_{k}:=\{i \leq$ $k: \nu_{i}$ is measure $\}$, and $\delta_{k}(x):=\sum_{i \in J_{k}} \varepsilon_{i} \nu_{i}(x)$. The weights $\varepsilon_{i}$ need to be computable and exponentially decreasing in $i$ and $\sum_{i=1}^{\infty} \varepsilon_{i} \leq 1$. We choose $\varepsilon_{i}=i^{-6} 2^{-i}$. Note the subtle and important fact that although the definition of $J_{k}$ is non-constructive, as a finite set of finite objects, $J_{k}$ is decidable (the program is unknowable for large $k$ ). Hence, $\delta_{k}$ is computable, since enumerable measures are computable.

$$
D(x)=\delta_{\infty}(x)=\sum_{i \in J_{\infty}} \varepsilon_{i} \nu_{i}(x)=\text { mixture of all computable measures. }
$$

In contrast to $J_{k}$ and $\delta_{k}$, the set $J_{\infty}$ and hence $D$ are neither enumerable nor co-enumerable. We also define the measures $\hat{\delta}_{k}(x):=\delta_{k}(x) / \delta_{k}(\epsilon)$ and $\hat{D}(x):=$ $D(x) / D(\epsilon)$. The following Proposition implies posterior convergence of $D$ to $\mu$ on $\mu$-random sequences.

Proposition 11 (Convergence of incomputable measure $\hat{D}$ ) Let $\mu$ be a computable measure with index $k_{0}$, i.e. $\mu=\nu_{k_{0}}$. Then for the incomputable measure $\hat{D}$ and the computable but non-constructive measures $\hat{\delta}_{k_{0}}$ defined above, the following holds:

$$
\begin{aligned}
& \text { i) } \quad \sum_{t=1}^{\infty} h_{t}\left(\hat{\delta}_{k_{0}}, \mu\right) \stackrel{ \pm}{\leq} 2 \ln 2 \cdot d_{\mu}(\omega)+3 k_{0} \\
& \text { ii) } \sum_{t=1}^{\infty} h_{t}\left(\hat{\delta}_{k_{0}}, \hat{D}\right) \stackrel{\times}{\leq} k_{0}^{7} 2^{k_{0}+d_{\mu}(\omega)}
\end{aligned}
$$

Combining $(i)$ and $(i i)$, using Lemma 9(i), we get $\sum_{t=1}^{\infty} h_{t}(\mu, \hat{D}) \leq c_{\omega} f\left(k_{0}\right)<\infty$ for $\mu$-random $\omega$, which implies $D\left(b \mid \omega_{<t}\right) \equiv \hat{D}\left(b \mid \omega_{<t}\right) \rightarrow \mu\left(b \mid \omega_{<t}\right)$. We do not know whether on-sequence convergence of the ratio holds. Similar bounds hold for $\hat{\delta}_{k_{1}}$ instead $\hat{\delta}_{k_{0}}, k_{1} \geq k_{0}$. The principle proof idea is to convert the expected bounds of Lemma 4 to individual bounds, using Lemma 10. The problem is that $\hat{D}$ is not computable, which we circumvent by joining with Lemma 9 , bounds on $\sum_{t} h_{t}\left(\hat{\delta}_{k-1}, \hat{\delta}_{k}\right)$ for $k=k_{0}, k_{0}+1, \ldots$

Proof. $(i)$ Let $H(\omega):=\sum_{t=1}^{\infty} h_{t}\left(\hat{\delta}_{k_{0}}, \mu\right) . \quad \mu$ and $\hat{\delta}_{k_{0}}$ are measures with $\hat{\delta}_{k_{0}} \geq \delta_{k_{0}} \geq$ $\varepsilon_{k_{0}} \mu$, since $\delta_{k}(\epsilon) \leq 1, \mu=\nu_{k_{0}}$ and $k_{0} \in J_{k_{0}}$. Hence, Lemma 4 applies and shows $\mathbf{E}_{\mu}\left[\exp \left(\frac{1}{2} H\right)\right] \leq \varepsilon_{k_{0}}^{-1 / 2}$. $H$ is well-defined and enumerable for $d_{\mu}(\omega)<\infty$, since $d_{\mu}(\omega)<$ 
$\infty$ implies $\mu\left(\omega_{1: t}\right) \neq 0$ implies $\hat{\delta}_{k_{0}}\left(\omega_{1: t}\right) \neq 0$. So $\mu\left(b \mid \omega_{1: t}\right)$ and $\hat{\delta}_{k_{0}}\left(b \mid \omega_{1: t}\right)$ are well defined and computable (given $\left.J_{k_{0}}\right)$. Hence $h_{t}\left(\hat{\delta}_{k_{0}}, \mu\right)$ is computable, hence $H(\omega)$ is enumerable. Lemma 10] then implies $\left.\exp \left(\frac{1}{2} H(\omega)\right) \stackrel{\times}{\leq} \varepsilon_{k_{0}}^{-1 / 2} \cdot 2^{K\left(\mu, H, \sqrt{\varepsilon_{k_{0}}}\right.}\right)+d_{\mu}(\omega)$. We bound

$$
K\left(\mu, H, \sqrt{\varepsilon}_{k_{0}}\right) \stackrel{ \pm}{\leq} K\left(H \mid \mu, k_{0}\right)+K\left(k_{0}\right) \stackrel{ \pm}{\leq} K\left(J_{k_{0}} \mid k_{0}\right)+K\left(k_{0}\right) \stackrel{ \pm}{\leq} k_{0}+2 \log k_{0}
$$

The first inequality holds, since $k_{0}$ is the index and hence a description of $\mu$, and $\varepsilon_{*}$ is a simple computable function. $H$ can be computed from $\mu, k_{0}$ and $J_{k_{0}}$, which implies the second inequality. The last inequality follows from $K\left(k_{0}\right) \stackrel{ \pm}{\leq} \log k_{0}$ and the fact that for each $i \leq k_{0}$ one bit suffices to specify (non)membership to $J_{k_{0}}$, i.e. $K\left(J_{k_{0}} \mid k_{0}\right) \stackrel{ \pm}{\leq} k_{0}$. Putting everything together we get

$$
H(\omega) \stackrel{ \pm}{\leq} \ln \varepsilon_{k_{0}}^{-1}+\left[k_{0}+2 \log k_{0}+d_{\mu}(\omega)\right] 2 \ln 2 \stackrel{ \pm}{\leq}(2 \ln 2) d_{\mu}(\omega)+3 k_{0} .
$$

(ii) Let $H^{k}(\omega):=\sum_{t=1}^{\infty} h_{t}\left(\hat{\delta}_{k}, \hat{\delta}_{k-1}\right)$ and $k>k_{0} . \delta_{k-1} \leq \delta_{k}$ implies

$$
\frac{\hat{\delta}_{k-1}(x)}{\hat{\delta}_{k}(x)} \leq \frac{\delta_{k}(\epsilon)}{\delta_{k-1}(\epsilon)} \leq \frac{\delta_{k-1}(\epsilon)+\varepsilon_{k}}{\delta_{k-1}(\epsilon)}=1+\frac{\varepsilon_{k}}{\delta_{k-1}(\epsilon)} \leq 1+\frac{\varepsilon_{k}}{\varepsilon_{O}},
$$

where $O:=\min \left\{i \in J_{k-1}\right\}=O(1)$. Note that $J_{k-1} \ni k_{0}$ is not empty. Since $\hat{\delta}_{k-1}$ and $\hat{\delta}_{k}$ are measures, Lemma 4 applies and shows $\mathbf{E}_{\hat{\delta}_{k-1}}\left[H^{k}\right] \leq \ln \left(1+\frac{\varepsilon_{k}}{\varepsilon_{O}}\right) \leq \frac{\varepsilon_{k}}{\varepsilon_{O}}$. Exploiting $\varepsilon_{k_{0}} \mu \leq \hat{\delta}_{k-1}$, this implies $\mathbf{E}_{\mu}\left[H^{k}\right] \leq \frac{\varepsilon_{k}}{\varepsilon_{O} \varepsilon_{k_{0}}}$. Lemma 10 then implies $H^{k}(\omega) \stackrel{x}{\leq} \frac{\varepsilon_{k}}{\varepsilon_{O} \varepsilon_{k_{0}}}$.

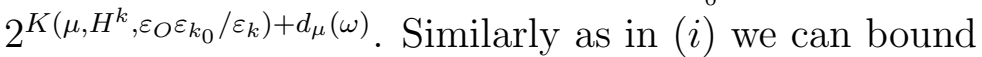

$$
\begin{gathered}
K\left(\mu, H^{k}, \varepsilon_{k_{0}} / \varepsilon_{O} \varepsilon_{k}\right) \stackrel{ \pm}{\leq} K\left(J_{k} \mid k\right)+K(k)+K\left(k_{0}\right) \stackrel{ \pm}{\leq} k+2 \log k+2 \log k_{0}, \quad \text { hence } \\
H^{k}(\omega) \stackrel{\times}{\leq} \frac{\varepsilon_{k}}{\varepsilon_{O} \varepsilon_{k_{0}}} \cdot k_{0}^{2} k^{2} 2^{k} c_{\omega} \stackrel{\times}{=} k_{0}^{8} 2^{k_{0}} k^{-4} c_{\omega}, \quad \text { where } \quad c_{\omega}:=2^{d_{\mu}(\omega)} .
\end{gathered}
$$

Chaining this bound via Lemma 9( $i i)$ we get for $k_{1}>k_{0}$ :

$$
\begin{aligned}
\sum_{t=1}^{n} h_{t}\left(\hat{\delta}_{k_{0}}, \hat{\delta}_{k_{1}}\right) & \leq \sum_{t=1}^{n} 3 \sum_{k=k_{0}+1}^{k_{1}}\left(k-k_{0}+1\right)^{2} h_{t}\left(\hat{\delta}_{k-1}, \hat{\delta}_{k}\right) \\
& \leq 3 \sum_{k=k_{0}+1}^{k_{1}} k^{2} H^{k}(\omega) \stackrel{\times}{\leq} 3 k_{0}^{8} 2^{k_{0}} c_{\omega} \sum_{k=k_{0}+1}^{k_{1}} k^{-2} \leq 3 k_{0}^{7} 2^{k_{0}} c_{\omega}
\end{aligned}
$$

If we now take $k_{1} \rightarrow \infty$ we get $\sum_{t=1}^{n} h_{t}\left(\hat{\delta}_{k_{0}}, \hat{D}\right) \stackrel{\times}{\leq} 3 k_{0}^{7} 2^{k_{0}+d_{\mu}(\omega)}$. Finally let $n \rightarrow \infty$.

The main properties allowing for proving $\hat{D} \rightarrow \mu$ were that $\hat{D}$ is a measure with approximations $\hat{\delta}_{k}$, which are computable in a certain sense. $\hat{D}$ is a mixture over all enumerable/computable measures and hence incomputable. 


\section{M.L.-Converging Enumerable Semimeasure $W$}

The next step is to enlarge the class of computable measures to an enumerable class of semimeasures, which are still sufficiently close to measures in order not to spoil the convergence result. For convergence w.p.1. we could include all semimeasures (Theorem [3). M.L.-convergence seems to require a more restricted class. Included non-measures need to be zero on long strings. We convert semimeasures $\nu$ to "quasimeasures" $\tilde{\nu}$ as follows:

$$
\tilde{\nu}\left(x_{1: n}\right):=\nu\left(x_{1: n}\right) \quad \text { if } \quad \sum_{y_{1: n}} \nu\left(y_{1: n}\right)>1-\frac{1}{n} \quad \text { and } \quad \nu\left(x_{1: n}\right):=0 \quad \text { else. }
$$

If the condition is violated for some $n$ it is also violated for all larger $n$, hence with $\nu$ also $\tilde{\nu}$ is a semimeasure. $\tilde{\nu}$ is enumerable if $\nu$ is enumerable. So if $\nu_{1}, \nu_{2}, \ldots$ is an enumeration of all enumerable semimeasures, then $\tilde{\nu}_{1}, \tilde{\nu}_{2}, \ldots$ is an enumeration of all enumerable quasimeasures. The for us important properties are that $\tilde{\nu}_{i} \leq \nu_{i}$-andif $\nu_{i}$ is a measure, then $\tilde{\nu}_{i} \equiv \nu_{i}$, else $\nu_{i}(x)=0$ for sufficiently long $x$. We define the enumerable semimeasure

$W(x):=\sum_{i=1}^{\infty} \varepsilon_{i} \tilde{\nu}_{i}(x), \quad$ andnote that $\quad D(x)=\sum_{i \in J} \varepsilon_{i} \tilde{\nu}_{i}(x)$ with $J:=\left\{i: \tilde{\nu}_{i}\right.$ is measure $\}$ with $\varepsilon_{i}=i^{-6} 2^{-i}$ as before.

Proposition 12 (Convergence of enumerable $W$ to incomputable $D$ ) For every computable measure $\mu$ and for $\omega$ being $\mu$-random, the following holds for $t \rightarrow \infty$ :

(i) $\frac{W\left(\omega_{1: t}\right)}{D\left(\omega_{1: t}\right)} \rightarrow 1, \quad$ (ii) $\frac{W\left(\omega_{t} \mid \omega_{<t}\right)}{D\left(\omega_{t} \mid \omega_{<t}\right)} \rightarrow 1, \quad$ (iii) $W\left(a \mid \omega_{<t}\right) \rightarrow D\left(a \mid \omega_{<t}\right) \quad \forall a \in \mathcal{X}$

The intuitive reason for the convergence is that the additional contributions of non-measures to $W$ absent in $D$ are zero for long sequences.

Proof. $(i)$

$$
D(x) \leq W(x)=D(x)+\sum_{i \notin J} \varepsilon_{i} \tilde{\nu}_{i}(x) \leq D(x)+\sum_{i=k_{x}}^{\infty} \varepsilon_{i} \tilde{\nu}_{i}(x),
$$

where $k_{x}:=\min _{i}\left\{i \notin J: \tilde{\nu}_{i}(x) \neq 0\right\}$. For $i \notin J, \tilde{\nu}_{i}$ is not a measure. Hence $\tilde{\nu}_{i}(x)=0$ for sufficiently long $x$. This implies $k_{x} \rightarrow \infty$ for $\ell(x) \rightarrow \infty$, hence $W(x) \rightarrow D(x) \forall x$. To get convergence in ratio we have to assume that $x=\omega_{1: n}$ with $\omega$ being $\mu$-random, i.e. $c_{\omega}:=\sup _{n} \frac{M\left(\omega_{1: n}\right)}{\mu\left(\omega_{1: n}\right)}=2^{d_{\mu}(\omega)}<\infty$.

$$
\Rightarrow \tilde{\nu}_{i}(x) \leq \nu_{i}(x) \leq \frac{1}{w_{\nu_{i}}} M(x) \leq \frac{c_{\omega}}{w_{\nu_{i}}} \mu(x) \leq \frac{c_{\omega}}{w_{\nu_{i}} \varepsilon_{k_{0}}} D(x)
$$


The last inequality holds, since $\mu$ is a computable measure of index $k_{0}$, i.e. $\mu=\nu_{k_{0}}=$ $\tilde{\nu}_{k_{0}}$. Inserting $1 / w_{\nu_{i}} \leq c^{\prime} \cdot i^{2}$ for some $c=O(1)$ and $\varepsilon_{i}$ we get $\varepsilon_{i} \tilde{\nu}_{i}(x) \leq \frac{c^{\prime} c_{\omega}}{\varepsilon_{k_{0}}} i^{-4} 2^{-i} D(x)$, which implies $\sum_{i=k_{x}}^{\infty} \varepsilon_{i} \tilde{\nu}_{i}(x) \leq \varepsilon_{x}^{\prime} D(x)$ with $\varepsilon_{x}^{\prime}:=\frac{2 c^{\prime} c_{\omega}}{\varepsilon_{k_{0}}} k_{x}^{-4} 2^{-k_{x}} \rightarrow 0$ for $\ell(x) \rightarrow \infty$. Inserting this into (8) we get

$$
1 \leq \frac{W(x)}{D(x)} \leq 1+\varepsilon_{x}^{\prime} \stackrel{\ell(x) \rightarrow \infty}{\longrightarrow} 1 \text { for } \mu \text {-random } x .
$$

(ii) Obvious from $(i)$ by taking a double ratio.

(iii) Let $a \in \mathcal{X}$. From $W(x a) \geq D(x a)(W \geq D)$ and $W(x) \leq\left(1+\varepsilon_{x}^{\prime}\right) D(x)(i)$ we get

$$
\begin{aligned}
W(a \mid x) & \geq\left(1+\varepsilon_{x}^{\prime}\right)^{-1} D(a \mid x) \geq\left(1-\varepsilon_{x}^{\prime}\right) D(a \mid x) \quad \forall a \in \mathcal{X}, \quad \text { and } \\
1-W(a \mid x) & \geq \sum_{b \neq a} W(b \mid x) \geq\left(1-\varepsilon_{x}^{\prime}\right) \sum_{b \neq a} D(b \mid x)=\left(1-\varepsilon_{x}^{\prime}\right)(1-D(a \mid x)),
\end{aligned}
$$

where we used in the second line that $W$ is a semimeasure and $D$ proportional to a measure. Together this implies $|W(a \mid x)-D(a \mid x)| \leq \varepsilon_{x}^{\prime}$. Since $\varepsilon_{x}^{\prime} \rightarrow 0$ for $\mu$-random $x$, this shows $(i i i) . h_{x}(W, D) \leq \varepsilon_{x}^{\prime}$ can also be shown.

Speed of convergence. The main convergence Theorem 8 now immediately follows from Propositions 11] and 12. We briefly remark on the convergence rate. Lemma [4 shows that $\mathbf{E}\left[\sum_{t} h_{t}(X, \mu)\right]$ is logarithmic in the index $k_{0}$ of $\mu$ for $X=M\left(\ln w_{k_{0}}^{-1} \stackrel{\times}{=} \ln k_{0}\right)$, but linear for $X=\left[W, D, \delta_{k_{0}}\right]\left(\ln \varepsilon_{k_{0}} \stackrel{\times}{=} k_{0}\right)$. The individual bounds for $\sum_{t} h_{t}\left(\hat{\delta}_{k_{0}}, \mu\right)$ and $\sum_{t} h_{t}\left(\hat{\delta}_{k_{0}}, \hat{D}\right)$ in Proposition 111 are linear and exponential in $k_{0}$, respectively. For $W \stackrel{M . L}{\longrightarrow} D$ we could not establish any convergence speed.

Finally we show that $W$ does not dominate all enumerable semimeasures, as the definition of $W$ suggests. We summarize all computability, measure, and dominance properties of $M, D, \hat{D}$, and $W$ in the following theorem:

Theorem 13 (Properties of $M, W, D$, and $\hat{D}$ )

(i) $M$ is an enumerable semimeasure, which dominates all enumerable semimeasures. $M$ is not computable and not a measure.

(ii) $\hat{D}$ is a measure, $D$ is proportional to a measure, both dominating all enumerable quasimeasures. $D$ and $\hat{D}$ are not computable and do not dominate all enumerable semimeasures.

(iii) $W$ is an enumerable semimeasure, which dominates all enumerable quasimeasures. $W$ is not itself a quasimeasure, is not computable, and does not dominate all enumerable semimeasures.

We conjecture that $D$ and $\hat{D}$ are not even approximable (limit-computable), but lie somewhere higher in the arithmetic hierarchy. Since $W$ can be normalized to an approximable measure M.L.-converging to $\mu$, and $D$ was only an intermediate quantity, the question of approximability of $D$ seems not too interesting. 


\section{Conclusions}

We investigated a natural strengthening of Solomonoff's famous convergence theorem, the latter stating that with probability 1 (w.p.1) the posterior of a universal semimeasure $M$ converges to the true computable distribution $\mu(M \stackrel{w \cdot p .1}{\longrightarrow} \mu)$. We answered partially negative the question of whether convergence also holds individually for all Martin-Löf (M.L.) random sequences $\left(\exists M: M_{\stackrel{M_{j}}{\rightarrow}}^{\rightarrow} \mu\right)$. We constructed random sequences $\alpha$ for which there exist universal semimeasures on which convergence fails. Multiplicative dominance of $M$ is the key property to show convergence w.p.1. Dominance over all measures is also satisfied by the restricted mixture $W$ over all quasimeasures. We showed that $W$ converges to $\mu$ on all M.L.-random sequences by exploiting the incomputable mixture $D$ over all measures. For $D \stackrel{M . L .}{\longrightarrow} \mu$ we achieved a (weak) convergence rate; for $W \stackrel{M . L .}{\longrightarrow} D$ and $W / D \stackrel{\text { M.L. }}{\longrightarrow} 1$ only an asymptotic result. The convergence rate properties w.p.1. of $D$ and $W$ are as excellent as for $M$.

We do not know whether $D / \mu \stackrel{M . L}{\longrightarrow} 1$ holds. We also don't know the convergence rate for $W \stackrel{M . L .}{\longrightarrow} D$, and the current bound for $D \stackrel{M . L .}{\longrightarrow} \mu$ is double exponentially worse than for $M \stackrel{w . p .1}{\longrightarrow} \mu$. A minor question is whether $D$ is approximable (which is unlikely). Finally there could still exist universal semimeasures $M$ (dominating all enumerable semimeasures) for which M.L.-convergence holds $(\exists M: M \stackrel{M . L .}{\longrightarrow} \mu$ ?). In case they exist, we expect them to have particularly interesting additional structure and properties. While most results in algorithmic information theory are independent of the choice of the underlying universal Turing machine (UTM) or universal semimeasure (USM), there are also results which depend on this choice. For instance, one can show that $\left\{(x, n): K_{U}(x) \leq n\right\}$ is tt-complete for some $U$, but not tt-complete for others [MP02]. A potential $U$ dependence also occurs for predictions based on monotone complexity [Hut03d]. It could lead to interesting insights to identify a class of "natural" UTMs/USMs which have a variety of favorable properties. A more moderate approach may be to consider classes $\mathcal{C}_{i}$ of UTMs/USMs satisfying certain properties $\mathcal{P}_{i}$ and showing that the intersection $\cap_{i} \mathcal{C}_{i}$ is not empty.

Another interesting and potentially fruitful approach to the convergence problem at hand is to consider other classes of semimeasures $\mathcal{M}$, define mixtures $M$ over $\mathcal{M}$, and (possibly) generalized randomness concepts by using this $M$ in Definition 5 , Using this approach, in Hut03b it has been shown that convergence holds for a subclass of Bernoulli distributions if the class is dense, but fails if the class is gappy, showing that a denseness characterization of $\mathcal{M}$ could be promising in general.

Acknowledgements. We want to thank Alexey Chernov for his invaluable help. 


\section{References}

[Hut03a] M. Hutter. Convergence and loss bounds for Bayesian sequence prediction. IEEE Transactions on Information Theory, 49(8):2061-2067, 2003.

[Hut03b] M. Hutter. On the existence and convergence of computable universal priors. In Proc. 14th International Conf. on Algorithmic Learning Theory (ALT-2003), volume 2842 of LNAI, pages 298-312, Berlin, 2003. Springer.

[Hut03c] M. Hutter. An open problem regarding the convergence of universal a priori probability. In Proc. 16th Annual Conf. on Learning Theory (COLT-2003), volume 2777 of $L N A I$, pages 738-740, Berlin, 2003. Springer.

[Hut03d] M. Hutter. Sequence prediction based on monotone complexity. In Proc. 16th Annual Conf. on Learning Theory (COLT-2003), Lecture Notes in Artificial Intelligence, pages 506-521, Berlin, 2003. Springer.

[Lev73] L. A. Levin. On the notion of a random sequence. Soviet Mathematics Doklady, 14(5):1413-1416, 1973.

[LV97] M. Li and P. M. B. Vitányi. An introduction to Kolmogorov complexity and its applications. Springer, 2nd edition, 1997.

[ML66] P. Martin-Löf. The definition of random sequences. Information and Control, 9(6):602-619, 1966.

[MP02] An. A. Muchnik and S. Y. Positselsky. Kolmogorov entropy in the context of computability theory. Theoretical Computer Science, 271(1-2):15-35, 2002.

[Sch02] J. Schmidhuber. Hierarchies of generalized Kolmogorov complexities and nonenumerable universal measures computable in the limit. International Journal of Foundations of Computer Science, 13(4):587-612, 2002.

[Sol64] R. J. Solomonoff. A formal theory of inductive inference: Part 1 and 2. Information and Control, 7:1-22 and 224-254, 1964.

[Sol78] R. J. Solomonoff. Complexity-based induction systems: comparisons and convergence theorems. IEEE Transaction on Information Theory, IT-24:422-432, 1978.

[VL00] P. M. B. Vitányi and M. Li. Minimum description length induction, Bayesianism, and Kolmogorov complexity. IEEE Transactions on Information Theory, 46(2):446-464, 2000.

[Vov87] V. G. Vovk. On a randomness criterion. Soviet Mathematics Doklady, 35(3):656660, 1987.

[ZL70] A. K. Zvonkin and L. A. Levin. The complexity of finite objects and the development of the concepts of information and randomness by means of the theory of algorithms. Russian Mathematical Surveys, 25(6):83-124, 1970. 